regulated gene expression in colon cancer systems of reducing complexity. Gene Expr. 2004;12(1):49-59.

13. Lopez de Silanes I, et al. Role of the RNA-binding protein $\mathrm{HuR}$ in colon carcinogenesis. Oncogene. 2003; 22(46):7146-7154

14. Lopez de Silanes I, Lal A, Gorospe M. HuR: posttranscriptional paths to malignancy. RNA Biol. 2005; 2(1):11-13

15. Rhee WJ, Ni CW, Zheng Z, Chang K, Jo H, Bao G. HuR regulates the expression of stress-sensitive genes and mediates inflammatory response in human umbilical vein endothelial cells. Proc Natl Acad Sci U S A. 2010;107(15):6858-6863

16. Yoo PS, et al. Tissue microarray analysis of 560 patients with colorectal adenocarcinoma: high expression of HuR predicts poor survival. Ann Surg
Oncol. 2009;16(1):200-207.

17. Denkert C, et al. Expression of the ELAV-like protein $\mathrm{HuR}$ is associated with higher tumor grade and increased cyclooxygenase- 2 expression in human breast carcinoma. Clin Cancer Res. 2004; 10(16):5580-5586.

18. Denkert C, et al. Overexpression of the embryoniclethal abnormal vision-like protein HuR in ovarian carcinoma is a prognostic factor and is associated with increased cyclooxygenase 2 expression. Cancer Res. 2004;64(1):189-195.

19. Gubin MM, et al. Overexpression of the RNA binding protein HuR impairs tumor growth in triple negative breast cancer associated with deficient angiogenesis. Cell Cycle. 2010;9(16):3337-3346.

20. Costantino CL, et al. The role of HuR in gemcitabi- ne efficacy in pancreatic cancer: HuR Up-regulates the expression of the gemcitabine metabolizing enzyme deoxycytidine kinase. Cancer Res. 2009; 69(11):4567-4572.

21. Atreya I, Neurath MF. Immune cells in colorectal cancer: prognostic relevance and therapeutic strategies. Expert Rev Anticancer Ther. 2008;8(4):561-572.

22. Lim SJ, Lee SH, Joo SH, Song JY, Choi SI. Cytoplasmic expression of HuR is related to cyclooxygenase-2 expression in colon cancer. Cancer Res Treat. 2009; 41(2):87-92.

23. Young LE, Sanduja S, Bemis-Standoli K, Pena EA, Price RL, Dixon DA. The mRNA binding proteins HuR and tristetraprolin regulate cyclooxygenase 2 expression during colon carcinogenesis. Gastroenterology. 2009;136(5):1669-1679.

\title{
Epoxyeicosatrienoic acids: a double-edged sword in cardiovascular diseases and cancer
}

\author{
Dingzhi Wang1 and Raymond N. DuBois ${ }^{1,2}$
}

${ }^{1}$ Department of Cancer Biology and 2Department of Gastrointestinal Medical Oncology, The University of Texas MD Anderson Cancer Center, Houston, Texas, USA.

\begin{abstract}
Epoxyeicosatrienoic acids (EETs) generated from arachidonic acid by cytochrome P450 (CYP) epoxygenases have beneficial effects in certain cardiovascular and kidney diseases. Hence, great efforts have been made to develop drugs targeting the EET pathway. Some of these agents are currently under evaluation in clinical trials for treatment of hypertension and diabetes. In this issue of the JCI, Panigrahy et al. evaluate in a systematic way the role of CYP epoxygenases and the metabolites they generate in cancer progression. Their findings, along with previous studies, raise concerns about using these drugs in humans.
\end{abstract}

High dietary fat intake is reported to be associated with several human diseases, including diabetes and heart disease. Moreover, epidemiologic and experimental observations support the hypothesis that high dietary fat intake is also a risk factor for cancers. However, the mechanisms underlying the link between high dietary fat intake and cancer progression are poorly understood. One factor thought to be involved is arachidonic acid (AA), a major component of animal fats that is primarily found in red meats, egg yolks, and organ meats. The bioactive lipids derived from AA play critical roles in cancer progression (1).

\section{Eicosanoid synthesis pathways}

AA is a polyunsaturated omega- 6 fatty acid that constitutes the phospholipid domain of most cell membranes. AA is liberated by cytoplasmic phospholipase $\mathrm{A}_{2}$ (cPLA2)

Conflict of interest: The authors have declared that no conflict of interest exists.

Citation for this article: J Clin Invest. 2012; 122(1):19-22. doi:10.1172/JCI61453.
(Figure 1). Free AA can be metabolized to eicosanoids through three major pathways: the prostaglandin-endoperoxide synthase/ cyclooxygenase (PTGS/COX) pathway, the lipoxygenase (LOX) pathway, and the cytochrome P450 (CYP) pathway. Prostanoids are the eicosanoids generated by the PTGS/COX pathway, while the LOX pathway generates leukotrienes and hydroxyeicosatetraenoic acids (HETEs). The CYP enzymes that convert AA into eicosanoids include CYP epoxygenase and CYP $\omega$-hydroxylase enzymes. CYP epoxygenases, such as members of the CYP2C and CYP2J families, metabolize AA to four biologically active epoxyeicosatrienoic acids (EETs) (5,6-EET, 8,9-EET, 11,12EET, and 14,15-EET). CYP $\omega$-hydroxylases, such as members of the CYP4A and CYP4F families, convert AA to HETEs. Among the members of the CYP2C and CYP2J families of CYP enzymes, CYP2J2, CYP2C8, and CYP2C9 are the predominant epoxygenase isoforms that convert AA into EETs. All EETs are then further metabolized to less active dihydroxyeicosatrienoic acids (DHETs) by soluble epoxide hydrolase (sEH).

\section{EET signaling in cardiovascular and kidney diseases}

EETs are produced primarily by endothelial cells, although they are also produced by other cell types, such as astrocytes and cardiomyocytes. Because EETs induce vasodilation and exert antiinflammatory effects in blood vessels in an autocrine manner (2), they can lower blood pressure, protect the myocardium and brain from ischemia, attenuate hypertension-induced renal damage, and reduce cigarette smokeinduced lung inflammation (3). Increasing evidence reveals that EETs govern these various biological functions by inducing endothelial cell proliferation, survival, and tube formation and stimulating renal epithelial cell proliferation and survival through multiple signaling pathways (4). Hence, great efforts have been made to develop drugs targeting these pathways. For example, 11,12-EET has been shown to improve coronary artery endothelial function when it is added to transplant preservation solutions (5). Moreover, an sEH inhibitor (AR9281) is currently under evaluation in phase II clinical trials as a treatment for patients with hypertension and type 2 diabetes on the basis of evidence that sEH inhibitors have beneficial effects in animal models of hypertension and cardiovascular diseases $(3,6)$. However, emerging evidence shows that EETs can promote cancer progression by directly promoting cancer cell proliferation, survival, migration, and invasion. In this issue of the JCI, 

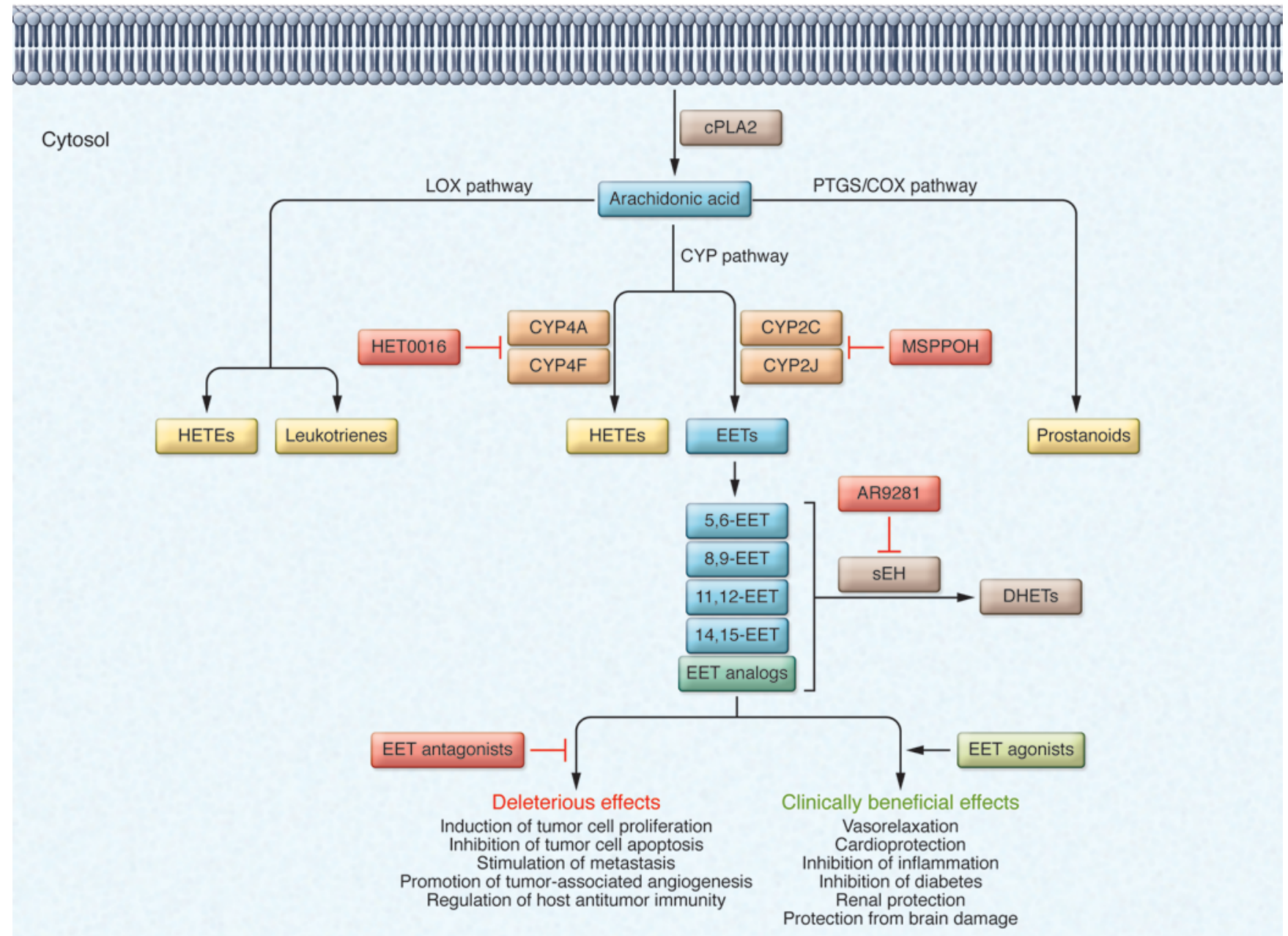

\section{Figure 1}

An overview of CYP epoxygenase pathways and how they can be modulated. AA is a polyunsaturated fatty acid that constitutes the phospholipid domain of most cell membranes. It is liberated from cellular membranes by cPLA2. Free AA can be metabolized through three major pathways: the PTGS/COX pathway, the LOX pathway, and the CYP pathway. In the CYP pathway, AA is converted to EETs and HETEs by CYP epoxygenases and CYP $\omega$-hydroxylases, respectively. The EETs are then further metabolized to less active DHETs by sEH. MSPPOH is a selective inhibitor of CYP epoxygenases, and HET0016 is a selective inhibitor of CYP $\omega$-hydroxylases. AR9281 is a selective inhibitor of sEH and is currently under evaluation in phase II clinical trials as a treatment for patients with hypertension and type 2 diabetes.

Panigrahy et al. report breakthroughs in our understanding of how EET signaling in the tumor microenvironment contributes to tumor growth and metastasis (7). These results raise concerns about using EET analogs and agonists as well as sEH inhibitors to treat cardiovascular diseases.

\section{EET signaling and cancer}

Although little is known about the role of EET signaling in cancer progression, emerging evidence indicates that CYP epoxygenases and the metabolites they generate are involved in tumor biology. CYP2J2 expression is elevated in human malignant solid tumors in esophageal, liver, breast, lung, and colorectal organs, and high levels of EETs have been detected in urine and blood samples obtained from patients with these cancers $(8,9)$. Interestingly, CYP2C9 is specifically expressed in the tumor-associated vasculature of human renal carcinomas (10). Moreover, hypoxia induces the expression of CYP2C8 and CYP2C9 (11). These results indicate that CYP epoxygenases may be involved in tumor-associated angiogenesis.

In vitro studies have shown that both overexpression of CYP2J2 in cancer cells and treatment of cancer cells with EETs stimulate cell proliferation, survival, migration, and invasion $(8,12)$. Further, in mouse xenograft models, overexpression of CYP2J2 in breast cancer cells promotes lung metastasis (12), whereas treatment with a selective inhibitor of CYP2J2 suppresses breast tumor growth and lung metastasis (13). Moreover, disruption of the Cyp $2 c 44$ gene of the xenograft recipient inhibits tumor growth and tumor-associated angiogenesis (10). Yet another study showed that preventive treatment of mice with CYP2J peptide inhibited tumor growth by activating host antitumor immunity at an initial stage of an implanted murine bladder tumor, whereas continuous treatment of mice with this peptide accelerated tumor growth by suppressing host antitumor 
immunity at an advanced stage (14). These findings indicate that CYP epoxygenases and EETs may play an important role in the tumor microenvironment.

Although EETs are well known to stimulate angiogenesis by promoting endothelial cell proliferation, survival, migration, and tube formation, surprisingly little research has directly addressed the question of how modification of EET signaling in endothelial cells affects neoplastic growth and metastasis. In this issue of the JCI, Panigrahy and colleagues present the first direct evidence showing that elevation of EET levels in endothelial cells leads to the promotion of tumor-associated angiogenesis and metastasis (7). They found that treatment of endothelial cells with 14,15-EET promoted primary tumor growth and metastasis. Similarly, elevation of EET levels by overexpression of either CYP2C8 or CYP2J2 in endothelial cells and by cell typenonspecific deletion of sEH also stimulated tumor growth and metastasis. By contrast, reduction of EET levels by overexpression of sEH in endothelial cells inhibited tumor growth and metastasis. Importantly, these investigators used a mouse model of prostate cancer in which the tumor develops spontaneously to confirm the results from their xenograft (subcutaneous and orthotopic) studies. Similarly, they found that treatment of mice with inhibitors of sEH accelerated tumor growth and metastasis. These data generated by Panigrahy and colleagues significantly extend our understanding of how EET signaling in the tumor microenvironment contributes to tumor growth and metastasis.

The data from human specimens and the in vitro and in vivo studies discussed here support the hypothesis that EETs may promote cancer progression by directly inducing cancer cell proliferation, survival, migration, and invasion and/or by changing the tumor microenvironment by influencing angiogenesis and immunosuppression in an autocrine and/or paracrine manner. This hypothesis has been tested in preclinical studies in which inhibitors of epoxygenase and EET antagonists were evaluated for their ability to inhibit tumor formation and growth (Figure 1). For example, treatment of glioblastoma-bearing rats with CYP epoxygenase inhibitors was found to attenuate tumor growth and tumor-associated angiogenesis (15). Similarly, the work reported in this issue of the JCI by Panigrahy et al. (7) showed for the first time that an EET antagonist could inhibit tumor growth and metastasis as well as prolong survival in several animal models. These in vivo data are consistent with a previous study in which an EET antagonist inhibited EET-induced prostate carcinoma cell migration and invasion in vitro (16). Collectively, the results discussed here not only raise concerns about developing $\mathrm{sEH}$ inhibitors as well as EET analogs and agonists for human use to treat cardiovascular diseases, but also support the rationale for developing EET antagonists and inhibitors of CYP epoxygenase enzymes as antitumor agents (Figure 1).

\section{EET downstream signaling pathways in cancer}

Although no EET receptor(s) have yet been clearly identified, EETs have been shown to bind to GPCRs $(17,18)$ and to facilitate binding activity of the PPAR/RXR heterodimer to a peroxisome proliferator response element $(19,20)$. Moreover, 14,15-EET induces EGFR transactivation in cancer cells in vitro (21). Indeed, EETs induce cancer cell proliferation via the EGFR/PI3K/Akt and EGFR/MAPK pathways and promote cancer cell survival through multiple pathways, including the TNF- $\alpha$ pathway and antioxidant enzymemediated pathways $(8,22)$. Moreover, prometastatic MMPs may mediate the effects of EETs on metastasis (12). The report by Panigrahy et al. (7) reveals that a VEGF signaling pathway is affected by EETs in endothelial cells. Furthermore, they found that VEGF signaling was required for EET-induced tumor-associated angiogenesis, which accelerated tumor growth and metastasis. However, it remains unclear whether EETs promote cancer progression by binding to cell-surface receptors and/or intracellular receptors such as nuclear receptors, with subsequent enhancement of cell proliferation, promotion of angiogenesis, inhibition of apoptosis, and stimulation of invasion/motility. Identification of specific EET receptors will be critical not only to further understanding of the molecular, cellular, and biological mechanisms underlying the involvement of EETs in malignant diseases, but also to enable the development of EET receptor-specific antagonists as antitumor agents.

\section{Summary}

CYP epoxygenases and the metabolites they generate, EETs, clearly have cardiovascular protective effects. However, the findings by Panigrahy et al. in this issue of the
JCI (7) and other published results (8-16) indicate that EETs also promote tumor growth and metastasis in some contexts. This warrants further investigation before sEH inhibitors as well as EETs and their analogs and agonists can be considered as therapies for cardiovascular disease. Clarifying this issue is of critical importance in order to avoid harmful effects in patients who may be considered for treatment with this class of drugs.

\section{Acknowledgments}

This work is supported, in part, by NIH MERIT award R37 DK47297, R01 DK 62112, NCI P01 CA77839, and CPRIT RP100960. We also thank the National Colorectal Cancer Research Alliance (NCCRA) for generous support (to R.N. DuBois)

Address correspondence to: Raymond N. DuBois, Departments of Gastrointestinal Medical Oncology and Cancer Biology, The University of Texas MD Anderson Cancer Center, Unit 1492, 1515 Holcombe Blvd., Houston, Texas 77030, USA. Phone: 713.745.4495; Fax: 713.792.6375; E-mail: rdubois@mdanderson.org.

1. Wang D, DuBois RN. Eicosanoids and cancer. Nat Rev Cancer. 2010;10(3):181-193.

2. Fleming I. Vascular cytochrome p450 enzymes: physiology and pathophysiology. Trends Cardiovasc Med. 2008;18(1):20-25.

3. Imig JD, Hammock BD. Soluble epoxide hydrolase as a therapeutic target for cardiovascular diseases. Nat Rev Drug Discov. 2009;8(10):794-805.

4. Xu X, Zhang XA, Wang DW. The roles of CYP450 epoxygenases and metabolites, epoxyeicosatrienoic acids, in cardiovascular and malignant diseases. Adv Drug Deliv Rev. 2011;63(8):597-609.

5. Yang Q, Zhang RZ, Yim AP, He GW. Effect of 11,12-epoxyeicosatrienoic acid as an additive to $\mathrm{St}$. Thomas' cardioplegia and University of Wisconsin solutions on endothelium-derived hyperpolarizing factor-mediated function in coronary microarteries: influence of temperature and time. Ann Thorac Surg. 2003;76(5):1623-1630.

6. Shen HC. Soluble epoxide hydrolase inhibitors: a patent review. Expert Opin Ther Pat. 2010; 20(7):941-956.

7. Panigrahy D, et al. Epoxyeicosanoids stimulate multiorgan metastasis and tumor dormancy escape in mice. J Clin Invest. 2012;122(1):178-191.

8. Jiang JG, et al. Cytochrome P450 2J2 promotes the neoplastic phenotype of carcinoma cells and is up-regulated in human tumors. Cancer Res. 2005; 65(11):4707-4715.

9. Chen C, et al. Cytochrome P450 2J2 is highly expressed in hematologic malignant diseases and promotes tumor cell growth. J Pharmacol Exp Ther. 2010; 336(2):344-355.

10. Pozzi A, et al. The anti-tumorigenic properties of peroxisomal proliferator-activated receptor alpha are arachidonic acid epoxygenase-mediated. J Biol Chem. 2010;285(17):12840-12850.

11. Michaelis UR, Fisslthaler B, Barbosa-Sicard E, Falck JR, Fleming I, Busse R. Cytochrome P450 epoxygenases $2 \mathrm{C} 8$ and $2 \mathrm{C} 9$ are implicated in hypoxia-induced endothelial cell migration and angiogenesis. 
J Cell Sci. 2005;118(pt 23):5489-5498.

12. Jiang JG, et al. Cytochrome p450 epoxygenase promotes human cancer metastasis. Cancer Res. 2007; 67(14):6665-6674.

13. Chen C, et al. Selective inhibitors of CYP2J2 related to terfenadine exhibit strong activity against human cancers in vitro and in vivo.J Pharmacol Exp Ther. 2009;329(3):908-918.

14. Homma S, et al. Antigenic stimulation with cytochrome P450 2J expressed in mouse hepatocellular carcinoma cells regulates host anti-tumour immunity. Clin Exp Immunol. 2009;156(2):344-352.

15. Zagorac D, Jakovcevic D, Gebremedhin D, Harder DR. Antiangiogenic effect of inhibitors of cytochrome $\mathrm{P} 450$ on rats with glioblas- toma multiforme. J Cereb Blood Flow Metab. 2008; 28(8):1431-1439.

16. Nithipatikom K, et al. Inhibition of carcinoma cell motility by epoxyeicosatrienoicacid(EET) antagonists. Cancer Sci. 2010;101(12):2629-2636.

17. Spector AA. Arachidonic acid cytochrome P450 epoxygenase pathway. J Lipid Res. 2009;50(suppl):S52-S56.

18 . Yang W, et al. Characterization of 14,15 -epoxyeicosatrienoyl-sulfonamides as 14,15-epoxyeicosatrienoic acid agonists: use for studies of metabolism and ligand binding. J Pharmacol Exp Ther. 2007; 321(3):1023-1031.

19. Liu Y, et al. The antiinflammatory effect of laminar flow: the role of PPARgamma, epoxyeicosatrienoic acids, and soluble epoxide hydrolase. Proc Natl Acad
Sci U S A. 2005;102(46):16747-16752.

20. Fang X, et al. 14,15-Dihydroxyeicosatrienoic acid activates peroxisome proliferator-activated receptor-alpha. Am J Physiol Heart Circ Physiol. 2006; 290(1):H55-H63.

21. Cheng LM, et al. The epoxyeicosatrienoic acidstimulated phosphorylation of EGF-R involves the activation of metalloproteinases and the release of HB-EGF in cancer cells. Acta Pharmacol Sin. 2010; 31(2):211-218

22. Liu L, et al. Epoxyeicosatrienoic acids attenuate reactive oxygen species level, mitochondrial dysfunction, caspase activation, and apoptosis in carcinoma cells treated with arsenic trioxide.J Pharmacol Exp Ther. 2011;339(2):451-463.

\title{
Alteration of hypothalamic cellular dynamics in obesity
}

\author{
Edward B. Lee ${ }^{1}$ and Rexford S. Ahima ${ }^{2}$
}

\begin{abstract}
${ }^{1}$ Department of Pathology and Laboratory Medicine and 2Department of Medicine, Division of Endocrinology, Diabetes and Metabolism, Perelman School of Medicine at the University of Pennsylvania, Philadelphia, Pennsylvania, USA.
\end{abstract}

\begin{abstract}
The number of people who suffer from obesity and one or more of its adverse complications is rapidly increasing. It is becoming clear that diet, exercise, and other lifestyle modifications are insufficient strategies to combat this growing problem. Greater understanding of the mechanisms controlling our desire to feed and our ability to balance energy intake with energy expenditure are key to the development of pharmacological approaches for treating obesity. Although great strides have been made in our understanding of how the hypothalamus regulates feeding and energy balance, much less is known about how obesity affects the structure of the hypothalamus. The authors of two papers in this issue of the JCI have addressed this issue by examining the effects of obesity on neurons and glia in the hypothalamus. These studies reveal that obesity may be in part due to hypothalamic injury, which leads to inflammation and reduced neurogenesis. These findings support the notion that obesity is a disease that affects multiple organs, including the brain, and that disruption of normal brain function leads to abnormal regulation of peripheral metabolism.
\end{abstract}

\section{Introduction}

As the number of people who are obese continues to rise so does the number of cases of obesity-associated morbidities, including type 2 diabetes, hypertension, heart disease, sleep apnea, and cancer. Diet, exercise, and other lifestyle modifications are important approaches to treating obesity. However, it is becoming clear that pharmacological approaches are also needed to combat this major healthcare problem. Thus far, pharmacological approaches to treating obesity have shown limited efficacy in the clinic and have been associated with serious side

Conflict of interest: The authors have declared that no conflict of interest exists.

Citation for this article: J Clin Invest. 2012; 122(1):22-25. doi:10.1172/JCI61562. effects. This is largely because our understanding of the complex biology of obesity is incomplete. For example, while great strides have been made in our understanding of how the brain controls our desire to feed, as well as the processes underlying the balancing of energy intake and expenditure, little is known about how the structure and organization of the brain are altered by obesity.

\section{Central regulation of peripheral metabolism}

The role of the hypothalamus in the regulation of feeding and energy balance was first highlighted by lesion studies in rodents ( 1 , 2 ). Although these classic studies proposing the existence of "feeding" and "satiety" centers in the hypothalamus lacked anatomic precision and were overly simplistic in their interpretation, the importance of the hypothalamus in the regulation of feeding and energy balance was subsequently highlighted by the discovery that hormone and peptide regulators of feeding and metabolism act on the hypothalamus as well as the brainstem and other areas of the brain (3). The adipose tissue-derived hormone leptin exerts its inhibitory effects on food intake by modulating the function of neurons in the arcuate nucleus (an aggregation of neurons in the medial basal region of the hypothalamus). Specifically, leptin suppresses neurons that release neuropeptide Y (NPY) and agouti-related peptide - neuropeptides that normally increase appetite - while stimulating neurons that release proopiomelanocortin (POMC) - the precursor of several neuropeptides, including some that suppress appetite (3). Leptin, ghrelin, and other hormones that control energy metabolism also affect synaptic plasticity - the structural connections of neurons in the hypothalamus and other areas of the brain (4-7).

A hallmark of obesity is the ability of adipose tissue to expand and undergo significant remodeling in order to fulfill its role as the major energy-storing organ. This involves coordinated responses among various cell types, including adipocyte precursors, blood vessels, and immune cells (8). Inflammation in obese adipose tissue is related to adipocyte death, accumulation of macrophages and other immune cells, and metabolic dysfunction (8). Recent stud- 